\title{
EQUIVALENCE BETWEEN MODIFIED SYMPLECTIC Gram-Schmidt AND Householder SR ALGORIthmS
}

\author{
A. Salam \\ L.M.P.A \\ Université du Littoral Côte d'Opale \\ 50 rue F. Buisson BP699, F-62228 Calais Cedex, France \\ salam@lmpa.univ-littoral.fr
}

The symplectic Gram-Schmidt (SGS) orthogonalization process is a crutial procedure for some important structure-preserving methods in linear algebra. The algorithm perfoms a factorization $A=S R$, where the ordered columns of the matrix $S$ form a symplectic basis of the range of $A$, and $R$ is $J$-upper triangular. There exist two versions of SGS, the classical (CSGS) and the modified (MSGS). Both are equivalent in exact arithmetic, but have different numerical behaviors. In this paper, a numerical equivalence is showed between the MSGS algorithm and Householder SR algorithm applied to an embedded matrix obtained from $A$ by adding two blocks of zeros in the middle and the bottom of the matrix $A$. The latter algorithm is based on transformations which are symplectic and rank-one modification of the identity (symplectic Householder transformations).

\section{References}

[1] Å. Björck, Numerics of Gram-Schmidt orthogonalization, Linear Algebra Appl. 197/198 (1994), 297-316.

[2] A. Salam, On theoretical and numerical aspects of symplectic GramSchmidt-like algorithms, Numer. Algo., 39 (2005), pp. 237-242.

[3] A. Salam and A. Elfarouk and E. Al-Aidarous, Symplectic Householder Transformations for a $Q R$-like decomposition, a Geometric and Algebraic Approaches, J. Comput. Appl. Math., 214 (2008), pp. 533-548. 